\title{
Evaluation of relationship between DiPS, NEWS and SIRS and 21-day mortality: A prospective observational study
}

\author{
Rebecca A Walford ${ }^{1}$, Timothy H Rainer ${ }^{2 *}$ and Chen Wen Ngua ${ }^{3}$ \\ ${ }^{1}$ Foundation Year 1 Doctor, Pinderfields Hospital, Aberford Rd, Wakefield WF1 4DG, England, UK \\ ${ }^{2}$ Professor of Emergency Medicine, Division of Population Medicine, University Hospital Wales, Heath Park, Cardiff, CF14 4XN, Wales, UK \\ ${ }^{3}$ Emergency Unit, Heath Park, Cardiff, CF14 4XN, Wales, UK
}

\begin{abstract}
Background: Sepsis is common and preventable mortality for septic shock is potentially high. Effective tools to improve the recognition, management and riskstratification of sepsis are needed. This study aimed to validate and compare current tools for detecting mortality in patients presenting to an emergency department (ED) with possible sepsis and septic shock in Cardiff.

Methods: This prospective observational study was conducted from $15^{\text {th }}$ to $28^{\text {th }}$ May 2017 in the ED of a tertiary university hospital in Cardiff, recruiting patients aged 18 years or older with sepsis. The inclusion criteria were adults with SIRS $\geq 2$ plus infection. Patients underwent a full ED work up. The primary outcome was 21-day mortality. Other outcomes were Intensive Care Unit (ICU) admission and hospital length of stay (LoS).

Results: 5116 patients were evaluated of whom 128 (2.5\%) consecutive patients had sepsis, and 8/128 (6.3\%) had septic shock. The 21-day mortality for sepsis was $8 / 128$ (6.3\%) and for septic shock was 3/8 (37.5\%). Septic shock diagnosis based on the Diagnostic Investigation and Prediction of Shock (DiPS) criteria had the strongest association with 21-day mortality (P-value 0.0038).
\end{abstract}

Discussion: The DiPS definition of Septic Shock was shown to capture more patients and to be a better predictor of mortality compared to the traditional SIRS definition. Larger prospective studies are needed for validation of the DiPS Septic Shock definition.

\begin{abstract}
Abbreviations: AUC: Area Under Curve; COPD: Chronic Obstructive Pulmonary Disease; dBP: Diastolic Blood Pressure; DiPs: Diagnostic Investigation and Prediction of Shock; ED: Emergency Department; GCS: Glasgow Coma Score; ICU: Intensive Care Unit; IQR: Interquartile Range; LoS: Length Of Stay; MAP: Mean Arterial Pressure; NEWS: National Early Warning Score; NIV: Non-invasive Ventilation; qSOFA: quick-SOFA; ROC: Receiver Operating Characteristic curve; sBP: Systolic Blood Pressure; SIRS: Systemic Inflammatory Response Syndrome; SSI: Signs \& Symptoms of infection; UK: United Kingdom
\end{abstract}

\section{Introduction}

The incidence of sepsis is increasing owing partly to the growing prevalence of chronic conditions in the aging population [1]. Septic shock is associated with high mortality. In the United Kingdom (UK), sepsis is estimated to be responsible for $8 \%$ of deaths, costing the NHS $£ 2.5$ billion [2].

Early identification and management are crucial in reducing mortality. The current gold-standard treatment for sepsis in the UK is completion of the Sepsis-Six bundle within an hour of recognition [3]. If completed timely, it is associated with superior patient outcomes, reduced length of stay (LoS) and reduced mortality [4,5]. Currently however there are several pitfalls in the recognition and management of sepsis. Its presentation is heterogeneous and may by subtle, and failure to recognise and respond to sepsis has been regularly reported. Furthermore, in 2011, a Quality Systems Assessment reported that $34 \%$ of clinical units did not have protocols for managing sepsis [4]. Necessary improvements have been highlighted as a national priority [5-7].

Although sepsis and septic shock are defined conceptually, producing an effective quantitative definition is not so straightforward [8]. Given the scarce supporting evidence for alternatives, definitions have remained largely unchanged for over two decades [7]. The traditional definition for sepsis, proposed in 1991, uses the host inflammatory response syndrome criteria (SIRS). This model risk stratifies patients into three categories: sepsis, severe sepsis and septic shock [7]. Limitations with this model are recognized. Furthermore, emerging insights into its pathophysiology have prompted a review of diagnostic criterion [8] Alternative tools have been suggested in the literature, differing predominantly by the cut-off values chosen.

The National Early Warning Score (NEWS) was developed based on the rationale that adverse events, such as cardiac arrests or

*Correspondence to: Timothy $\mathrm{H}$ Rainer, Division of Population Medicine, University Hospital Wales, Heath Park, Cardiff, CF14 4XN, UK, E-mail: thrainer@cuhk.edu.hk

Key words: emergency department, infection, National Early Warning Score (NEWS), sepsis, septic shock, systemic inflammatory response syndrome (SIRS)

Received: October 09, 2018; Accepted: October 25, 2018; Published: October 29,2018 
admissions to high dependency unit, are commonly preceded by deranged physiological parameters [9]. NEWS scoring provides an appropriate framework for risk-stratification, and when coupled with early intervention, can help prevent deterioration of patients [10]. In the context of sepsis it facilitates early recognition and appropriate escalation [11].

A recently introduced method for evaluating patients with possible sepsis outside the Intensive Care Unit (ICU) is quick-sOFA (qSOFA). A meta-analysis found that qSOFA was marginally superior to SIRS for predicting in-hospital mortality, but was inferior for sepsis diagnosis due to its poorer sensitivity [12].

A new tool for classifying emergency department (ED) shock, the Diagnostic Investigation and Prediction of Shock (DiPS) tool, was validated in patients presenting to an ED in Hong Kong [13]. It did not focus exclusively on patients with possible sepsis and did not compare common risk-stratification tools. However, given its more encompassing definition, compared to SIRS septic shock, it has the potential for greater sensitivity.

This study aimed to validate and compare current tools for detecting mortality in patients presenting to an ED with sepsis or septic shock in Cardiff. These included the traditional SIRs scoring system, NEWS and the DiPs Septic Shock definition.

\section{Methods}

In this prospective quality improvement project, the need for ethical approval and consent for quality improvement projects is not required in Cardiff University and Cardiff and Vale University Health Board Clinical R\&D Departments.

\section{Inclusion and Exclusion Criteria}

Adult patients aged $\geq 18$ years, attending any area in the ED, with SIRS $\geq 2$ and infection were included. Patients with trauma, pregnancy, or who did not have a SIRS evaluation because of a minor injury were not included.

\section{Definitions}

An online database facilitated collection of a minimum dataset for each patient. Demographic data included postcode, age, sex, comorbidity and premorbid status. Clinical data included respiratory rate, heart rate, blood pressure, oxygen saturation, temperature and AVPU status. These measurements were used to calculate NEWS. Glasgow Coma Score was also recorded. Patients underwent a full ED work up. Investigations included full blood count, serum creatinine, urea and electrolytes, blood gases, blood glucose, clotting screen, International Normalised Ratio, liver function tests, blood culture, chest-X-ray and electrocardiogram. Therapeutic management details included time of initial antibiotic treatment, choice of antibiotic, volume of fluid resuscitation in the first six hours, use of noradrenaline and use of steroids. Date and time of presentation, time of triage, time first seen by a doctor and time transferred out of ED, triage category (Appendix) and the area in which the patient was predominantly managed were also recorded. Sepsis-Six compliance was assessed, including use of the Sepsis-Six form, documented measurement of urine output and lactate, administration of antibiotics within one hour, administration of intravenous fluids and oxygen, and taking blood cultures.

Patients were classified as having 'sepsis' if any two of the following Signs \& Symptoms of infection (SSI) criteria were present alongside presumed or proven infection, namely temperature $<36$ or $>38 \mathrm{C}$; heart rate $>90$ bpm; white cell count $>12$ or $<4 \times 109 / \mathrm{L}$; respiratory rate $\geq 20$ / min; acutely altered mental state; and hypoglycaemia in the absence of diabetes [14].

Patients were classified as 'SIRS septic shock' if both of the following were present alongside presumed or proven infection: evidence of an 'abnormal' blood pressure defined as either a SBP $<90 \mathrm{mmHg}$ or a mean arterial pressure (MAP) $<65 \mathrm{mmHg}$; and evidence of grossly abnormal acid-base status defined as a lactate level $\geq 4.0 \mathrm{mmol} / \mathrm{L}$ [14].

Patients were classified as 'DiPS septic shock' if any one of the following were present alongside presumed or proven infection: evidence of an 'abnormal' blood pressure defined as either a SBP $<90$ $\mathrm{mmHg}$ or a MAP $<65 \mathrm{mmHg}$; or evidence of grossly abnormal acidbase status defined as a lactate level $\geq 4.0 \mathrm{mmol} / \mathrm{L}$, or a base deficit of $\leq$ $-5 \mathrm{mEq} / \mathrm{L}$, or a $\mathrm{pH} \leq 7.1[13]$.

The primary outcome was 21-day mortality. Secondary outcomes were ICU admission, ICU LoS and hospital LoS.

\section{Statistical Analysis}

The data was analysed using MedCalc version 15.8 (MedCalc Software, Mariakerke, bvba, Belgium) and Microsoft Excel (Microsoft ${ }^{\circ}$ Excel $^{\circledR}$ for Mac 2011 version 14.7.2, Microsoft Corporation, Santa Rosa, California, USA) to calculate medians, means, standard deviations, interquartile ranges and confidence intervals. Chi squared and Fisher tests were also used. A P $\leq 0.05$ was considered to be significant.

\section{Results}

Between $15^{\text {th }}$ and $28^{\text {th }}$ May 2017, 5116 patients attended the ED of whom $128(2.5 \%)$ cases were recruited. Figure 1 illustrates the case selection process and flow of patients with sepsis through the ED. The incidence of sepsis was 25 per 1000 patient attendances. Eight of the $128(6.3 \%)$ septic patients were classified as having septic shock based on the SIRS criteria, whilst 47 (36.7\%) had septic shock based on DiPS criteria. The incidence of SIRS septic shock was 1.6 per 1000 patient attendances. The majority ( $\mathrm{n}=87,68.0 \%)$ was admitted to the wards. Four patients $(3.1 \%)$ were admitted to ICU. The 21 -day mortality rate for sepsis was $6.3 \%(8 / 128)$ and for septic shock was $37.5 \%(3 / 8)$. The average hospital $\operatorname{LoS}$ was 8.1 days \pm 10.7 days.

\section{Baseline Data}

Table 1 shows the baseline characteristics of the study patients, whilst Table 2 shows their baseline investigations. The majority of patients were elderly and had significant comorbidities but lived independently. Twenty-nine percent of cases came from deprived areas in Wales. In the majority of patients, the NEWS showed deterioration from when they first presented.

\section{NEWS, SIRS score, SIRS septic shock definition and DiPS septic shock definition as predictors of mortality}

As shown in Table 3, 47 cases (36.7\%) were classified to have Septic Shock based on DiPS definition. DiPS showed the strongest correlation with 21-day mortality, with an odds ratio of 14.0 (P-value 0.0151 ). This was closely followed by the more selective SIRS Septic Shock definition, which only included 8 septic shock cases (3.7\%). Five of these patients died by day-21 (odds ratio 13.8, P-value 0.0023 ). Cases were also grouped based on a NEWS $\geq 7$ and SIRs $\geq 3$. The odds of death by day- 21 in these groups were 7.2 (P-value 0.068 ) and 1.4 (P-value 0.414 ) respectively.

The association between each parameter of DiPS septic shock and 21-day mortality are shown in Table 4 . A lactate $\geq 4.0 \mathrm{mmol} / \mathrm{L}$ had the strongest association with 21 -day mortality (P-value $<0.0001)$, followed by base deficit (P-value 0.002) and sBP (P-value 0.012). 
Table 1. Baseline Characteristics ( $\mathrm{N}=128)$

\section{Variable}

Age -years -Median (IQR)

Male sex - no. $(\%)$

Index of deprivation - \% - Median (IQR) ${ }^{*}$

Past medical history/ comorbidity - no. (\%)

Chronic Obstructive Lung Disease - no. (\%)

Hypertension - no. (\%)

Diabetes Mellitus - no. (\%)

Other chronic lung disease - no. (\%)

Malignancy - no. (\%)

Ischemic heart disease - no. (\%)

Heart failure - no. (\%)

Hyperlipidaemia - no. (\%)

Chronic Kidney Disease - no. (\%)

Premorbid status

Independent - no. (\%)

Partially dependent - no. (\%)

Fully dependent - no. (\%)

Medication

Proton pump inhibitor - no. (\%)

Antihypertensive-no. (\%)

Statin - no. (\%)

Antidepressant - no. (\%)

Oral hypoglycaemic agent - no. (\%)

Immunosuppressant (including steroids) - no. (\%)

Insulin - no. (\%)

SIRS Score on Admission

$0-$ no. $(\%)$

$1-$ no. $(\%)$

$2-$ no. $(\%)$

3 - no. $(\%)$

4 - no. $(\%)$

\section{Observations}

Systolic Blood Pressure (sBP) $-\mathrm{mmHg}-$ Median (IQR)

Diastolic Blood Pressure (dBP) - mmHg - Median (IQR)

Mean Arterial Pressure (MAP) - mmHg - Median (IQR)

Pulse Pressure $-\mathrm{mmHg}-$ Median (IQR)

Heart Rate - bmp - Median (IQR)

Respiratory rate - bmp - Median (IQR)

Temperature $-{ }^{\circ} \mathrm{C}-$ Median (IQR)

Oxygen Saturations $\left(\mathrm{SaO}_{2}\right)-$ Median (IQR)

$\mathrm{FiO}_{2}-\%$ - Median (IQR)

Glasgow Coma Score - Median (IQR)

\section{NEWS}

0 - no. (\%)

1-3-no. (\%)

4-6-no. (\%)

7-9- no. (\%)

10-12-no. (\%)

13-18-no. (\%)

Triage Category

1 -no. (\%)

$2-$ no. $(\%)$
Numbers

$65(41.8-78.0)$

$68(53)$

$29(9.0-73.1)$

$112(87.5)$

$30(23.4)$

29 (22.7)

25 (19.5)

$20(15.6)$

$18(14.1)$

15 (11.7)

$10(7.8)$

$6(4.7)$

5 (3.9)

86 (67.2)

24 (18.8)

18 (14.1)

41 (32.0)

41 (32.0)

35 (27.3)

31 (24.2)

21 (16.4)

$16(12.5)$

2 (1.6)

\section{$0(0)$ \\ $0(0)$ \\ 73 (57.0) \\ 40 (31.3) \\ 15 (11.7)}

\begin{tabular}{c|c|} 
& \multicolumn{1}{c}{$15(11.7)$} \\
First & \\
126 & \\
$(105.8-139.0)$ & \\
74 &
\end{tabular}

$(59.0-82.0)$

90

(77.5 - 100.6)

49

$(39.0-62.0)$

105

$(94.0-119.0)$

22

(21.5-24.0)

37.6

(37.0-38.5)

96

$(93.0-98.0)$

21

$(21.0-21.0)$

15

$(15.0-15.0)$

First

$0(0)$

$41(32.0)$

51 (39.8)

$26(20.3)$

$7(5.5)$

3 (2.3)

Worst

107

$(92.0-128.0)$

63

$(51.0-76.0)$

76

$(64.3-93.3)$

42

(34.8-56.3)

99

$(87.8-112.8)$

20

(18.0-24.0)

37.4

(36.6-38.2)

97

$(95.0-98.0)$

21

$(21.0-28.0)$

15

$(15.0-15.0)$

Worst

$0(0)$

2 (1.6)

60 (46.9)

41 (32.0)

19 (14.8)

$6(4.7)$

$33(25.8)$

43 (33.6) 


\begin{tabular}{|c|c|}
\hline $3-$ no. $(\%)$ & $29(22.7)$ \\
\hline $4-$ no. (\%) & $23(18.0)$ \\
\hline \multicolumn{2}{|l|}{ Source of Infection } \\
\hline Respiratory - no. (\%) & $61(47.7)$ \\
\hline Urinary tract - no. $(\%)$ & $27(21.1)$ \\
\hline Abdomen - no. (\%) & $19(14.8)$ \\
\hline Skin/joint - no. (\%) & $13(10.2)$ \\
\hline Other $^{* 2}-$ no. (\%) & $7(5.5)$ \\
\hline Unknown - no. (\%) & $6(4.7)$ \\
\hline \multicolumn{2}{|l|}{ Acquired From } \\
\hline Community & $121(94.5)$ \\
\hline Nosocomial $^{* 3}$ & $7(5.5)$ \\
\hline
\end{tabular}

"calculated using Welsh Index of Multiple Deprivation (WIMD) 2014, 10\% indicates the local area is in the ' $10 \%$ most deprived areas in Wales' [15].

"2tonsillitis, Pelvic Inflammatory Disease (PID), sinusitis

${ }^{* 3}$ recent discharge within $72 \mathrm{~h}$.

Table 2. Baseline Investigations $(\mathrm{N}=128)$

\begin{tabular}{|c|c|}
\hline Variable & Numbers \\
\hline \multicolumn{2}{|l|}{ Electrocardiogram $(\mathrm{N}=108)$} \\
\hline Sinus rhythm - no. (\%) & $87(80.5 \%)$ \\
\hline Atrial fibrillation - no. (\%) & $17(15.7 \%)$ \\
\hline Ventricular rate - Median (IQR) & $100(99-114)$ \\
\hline \multicolumn{2}{|l|}{ Blood Tests } \\
\hline Haemoglobin - g/dL - Median (IQR) & $133.0(117.0-146.0)$ \\
\hline White Cell Count - /L - Median (IQR) & $13.0(9.5-15.0)$ \\
\hline Platelet - /L - Median (IQR) & $236(184.0-306.0)$ \\
\hline Neutrophils - /L - Median (IQR) & $10.0(6.73-12.0)$ \\
\hline Lymphocytes - /L - Median (IQR) & $1.05(0.60-1.70)$ \\
\hline Haematocrit - g/dL-Median (IQR) & $0.40(0.36-0.43)$ \\
\hline Creatinine $-\mu \mathrm{mol} / \mathrm{L}-$ Median $(\mathrm{IQR})$ & $76.0(67.0-103.5)$ \\
\hline Baseline creatinine ${ }^{*}-\mu \mathrm{mol} / \mathrm{L}-$ Median (IQR) & $71.0(59.5-83.5)$ \\
\hline Urea - mmol/L- Median (IQR) & $4.9(3.9-7.4)$ \\
\hline Sodium - mmol/L - Median (IQR) & $138.0(135.5-140.0)$ \\
\hline Potassium - mmol/L - Median (IQR) & $4.10(3.78-4.30)$ \\
\hline Serum Glucose - mmol/L - Median (IQR) & $6.9(6.3-9.1)$ \\
\hline Bilirubin - $\mu \mathrm{mol} / \mathrm{L}$ - Median (IQR) & $10.0(7.0-16.0)$ \\
\hline Alanine Transaminase - IU/1 - Median (IQR) & $21.0(14.0-35.0)$ \\
\hline Serum Albumin - g/L - Median (IQR) & $33.0(29.0-36.0)$ \\
\hline Prothrombin time - seconds - Median (IQR) & $13.6(12.5-15.1)$ \\
\hline Partial Thromboplastin Time (PTT) - seconds - Median (IQR) & $29.8(27.4-33.7)$ \\
\hline C-Reactive Protein - mg/L - Median (IQR) & $60.0(26.0-130.0)$ \\
\hline \multicolumn{2}{|l|}{ Venous Blood Gas } \\
\hline $\mathrm{pH}-$ Median (IQR) & $7.42(7.37-7.45)$ \\
\hline Partial Pressure of Oxygen $\left(\mathrm{PaO}_{2}\right)-\mathrm{mmHg}-$ Median (IQR) & $5.64(3.73-8.15)$ \\
\hline Partial Pressure of Carbon Dioxide $\left(\mathrm{PaCO}_{2}\right)-\mathrm{mmHg}-$ Median (IQR) & $5.06(4.5-6.0)$ \\
\hline Base deficit - mmol/ L-Median (IQR) & $0.70(-2.3-2.65)$ \\
\hline Bicarbonate - mmol/L - Median (IQR) & $24.20(21.85-26.10)$ \\
\hline Lactate - mmol/L - Median (IQR) & $1.60(0.95-2.75)$ \\
\hline
\end{tabular}

"pre-admission baseline creatinine

Table 3. Comparing SIRS and DiPS Septic Shock Definitions and NEWS and SIRS scores as predictors of 21-day mortality

\begin{tabular}{|c|c|c|c|c|c|c|c|}
\hline & & \multicolumn{2}{|c|}{ 21-Day Mortality - no. } & \multirow{2}{*}{ Odds Ratio (95\% CI) } & \multirow{2}{*}{$\operatorname{Sx}(1 \%)$} & \multirow{2}{*}{$\underset{(1 \%)}{S p}$} & \multirow{2}{*}{ P-value } \\
\hline & & Survived $(\mathrm{N}=120)$ & $\operatorname{Died}(\mathrm{N}=8)$ & & & & \\
\hline \multirow{2}{*}{ DiPS Septic Shock } & Yes & 40 & 7 & \multirow{2}{*}{$\begin{array}{c}14.0 \\
(1.7-117.7)\end{array}$} & \multirow{2}{*}{88} & \multirow{2}{*}{67} & \multirow{2}{*}{$0.0151^{*}$} \\
\hline & No & 80 & 1 & & & & \\
\hline \multirow{2}{*}{ SIRS Septic Shock } & Yes & 5 & 3 & \multirow{2}{*}{$\begin{array}{c}13.8 \\
(2.5-74.7)\end{array}$} & \multirow{2}{*}{38} & \multirow{2}{*}{96} & \multirow{2}{*}{$0.0023^{*}$} \\
\hline & No & 115 & 5 & & & & \\
\hline \multirow{2}{*}{ NEWS $>6$} & Yes & 59 & 7 & \multirow{2}{*}{$\begin{array}{c}7.2 \\
(0.9-60.6)\end{array}$} & \multirow{2}{*}{88} & \multirow{2}{*}{51} & \multirow{2}{*}{0.0680} \\
\hline & No & 61 & 1 & & & & \\
\hline \multirow{2}{*}{ SIRS $>2$} & Yes & 51 & 4 & \multirow{2}{*}{$\begin{array}{c}1.4 \\
(0.3-5.7)\end{array}$} & \multirow{2}{*}{50} & \multirow{2}{*}{58} & \multirow{2}{*}{0.414} \\
\hline & No & 69 & 4 & & & & \\
\hline \multicolumn{7}{|c|}{$\begin{array}{l}\text { "results are statistically significant }(\mathrm{P}-\mathrm{value}<0.05) \\
\text { Sx }- \text { sensitivity }\end{array}$} & \\
\hline
\end{tabular}


Table 4. DiPS Septic Shock parameters as predictors of 21-day mortality

\begin{tabular}{|c|c|c|c|c|c|c|}
\hline \multirow{2}{*}{\multicolumn{2}{|c|}{ DiPS Septic Shock Parameters }} & \multicolumn{2}{|c|}{ 21-Day Mortality } & \multirow{4}{*}{$\begin{array}{c}\begin{array}{c}\mathbf{S x}^{* k} \\
(\%)\end{array} \\
19\end{array}$} & \multirow{4}{*}{$\begin{array}{l}\mathbf{S p}^{* *} \\
(\%)\end{array}$} & \multirow{4}{*}{$\begin{array}{r}\text { P-value } \\
0.012^{*}\end{array}$} \\
\hline & & \multirow{3}{*}{$\begin{array}{c}\text { Survived }(\mathbf{N}=\mathbf{1 2 0}) \\
25 \\
95\end{array}$} & \multirow{3}{*}{$\begin{array}{c}\text { Died }(\mathbf{N}=\mathbf{8}) \\
6 \\
2\end{array}$} & & & \\
\hline \multirow{2}{*}{$\mathrm{sBP}(\mathrm{mmHg})$} & $<90$ & & & & & \\
\hline & $>90$ & & & & & \\
\hline \multirow{2}{*}{ MAP (mmHg) } & $<65$ & 29 & 4 & \multirow{2}{*}{12} & \multirow{2}{*}{96} & \multirow{2}{*}{0.102} \\
\hline & $>65$ & 91 & 4 & & & \\
\hline \multirow{3}{*}{ Lactate $(\mathrm{mmol} / \mathrm{L})$} & $\geq 4.0$ & 8 & 5 & \multirow{3}{*}{63} & \multirow{3}{*}{93} & \multirow{3}{*}{$<0.0001^{*}$} \\
\hline & $1.5-<4.0$ & 74 & 2 & & & \\
\hline & $<1.5$ & 38 & 1 & & & \\
\hline \multirow{3}{*}{$\mathrm{pH}$} & $\leq 7.1$ & 1 & 0 & \multirow{3}{*}{0} & \multirow{3}{*}{99} & \multirow{3}{*}{0.841} \\
\hline & $7.1-7.3$ & 4 & 0 & & & \\
\hline & $>7.3$ & 115 & 8 & & & \\
\hline Base deficit (mEq/L) & $\leq-5$ & 6 & 3 & 38 & 95 & $0.002^{*}$ \\
\hline \multicolumn{7}{|c|}{$\begin{array}{l}\text { Sx }- \text { sensitivity } \\
\mathrm{Sp} \text { - specificity } \\
{ }^{* *} \text { Where there are three groups, the lower two have }\end{array}$} \\
\hline
\end{tabular}

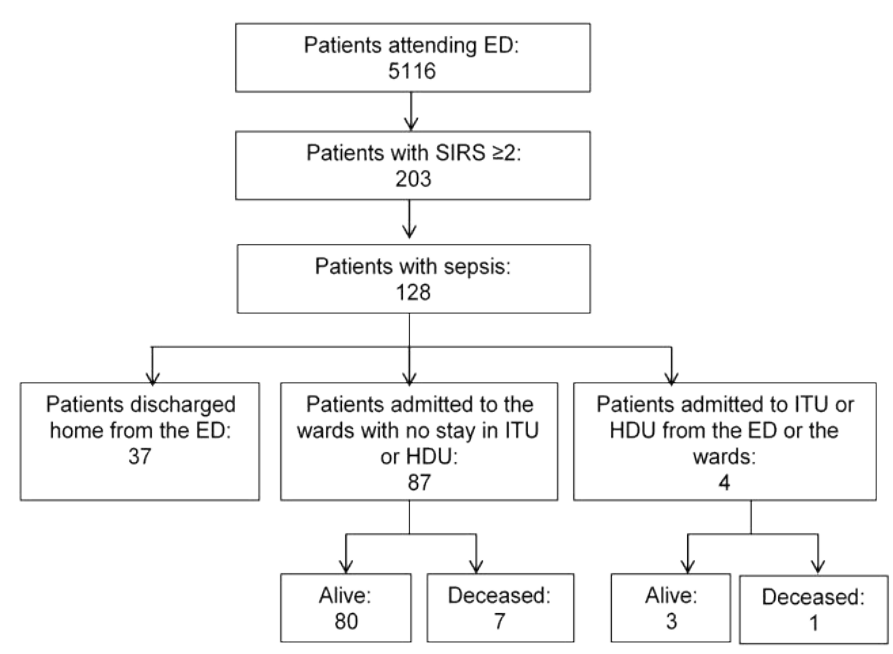

Figure 1. Flow of septic patients through the ED at University Hospital of Wales

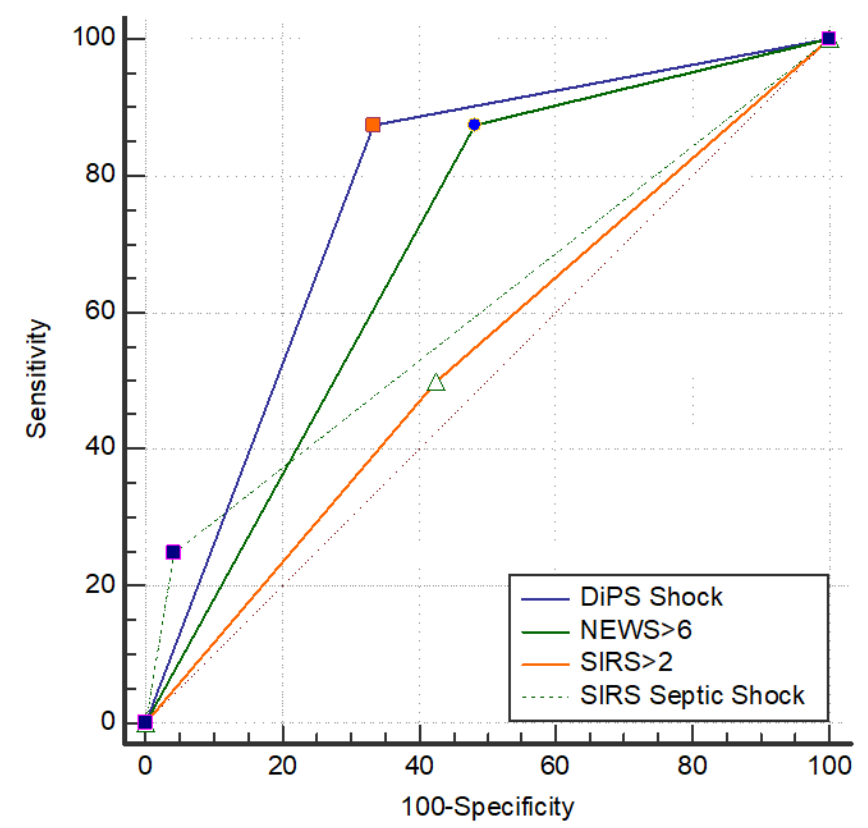

Figure 2. Receiver Operating Characteristic curve for NEWS and SIRS scores and SIRs and DiPS Septic Shock definitions
The validity of the four definitions are shown graphically in Figure 2. DiPS septic shock definition generated the greatest area under the Receiver Operating Characteristic curve (ROC) (AUC 0.771), followed by NEWS $\geq 7$ (AUC 0.696), then SIRS septic shock (AUC 0.604) and lastly SIRS $\geq 3$ (AUC 0.534). These values were not statistically significant, however the apparent trend is consistent with the statistically significant results shown in Table 2.

\section{Discussion}

This prospective study confirms that in a heterogenous group of $5116 \mathrm{ED}$ attendees, sepsis is a relatively common condition with septic shock being associated with high mortality and morbidity. The incidence of sepsis was 25 per 1000 patient attendances and the incidence of SIRS septic shock was 1.6 per 1000 patient attendances.

The demographic and clinical features of the study patients are consistent with the literature. In accordance with the already known high risk groups [16], the study cohort had a median age of 65 (41.878.0), with $14 \%(n=18)$ being fully dependent, $87.5 \%(n=112)$ having a comorbidity and $12.5 \%(n=16)$ being on an immunosuppressant. Kang, et al. (2011) showed that pulmonary disease, malignancy and liver disease were the strongest risk factors for septic shock, and strong predictors of mortality [17]. Pulmonary disease was the most common comorbidity in our study (39\%, Table 1), with Chronic Obstructive Pulmonary Disease (COPD) accounting for the majority [17]. Consistent with previous studies, respiratory tract infections were the most common site of infection [18].

Previous guidelines used SIRS to define sepsis and septic shock. The theories behind these definitions have been criticized as out-of-date and irrelevant. However a recent meta-analysis suggests the proposed replacement-qSOFA-may add little advantage in prognosis and none in detection [12]. Therefore, our study evaluated NEWS and DiPS as potential alternatives.

DiPS septic shock and all its individual parameters, except $\mathrm{pH}$ and MAP, correlated with 21-day mortality. MAP has been well reported to be independently associated with ICU admission and hospital mortality for patients with Septic Shock [19]. The lack of association in this study may be due to inadequate sample size. The prognostic role of low $\mathrm{pH}$ is unclear in the literature. For example, Goodhart, et al. (2015) found that low $\mathrm{pH}$ was associated with higher mortality in patients with pneumonia treated with non-invasive ventilation (NIV) but not in patients with COPD treated with NIV [20]. Further studies are needed to define the relevance of $\mathrm{pH}$ as a prognostic indicator. 
The SIRS septic shock definition captures a subset of patients defined under the DiPS septic shock definition. This subset was also associated with poorer prognosis. DiPS Septic Shock definition generated the greatest area under the ROC curve suggesting it had a superior combination of sensitivity and specificity. Although this result was not statistically significant, it highlights the need to re-evaluate the current definitions of Septic Shock that is advocated in the guidelines.

NEWS is known to be a strong predictor of mortality. It has been adopted as a generic screening and assessment risk-stratification tool throughout the UK [21]. Correspondingly in this study, NEWS $\geq 7$ was significantly associated with worse 21 -day mortality. SIRS score $\geq 3$ was not shown to be associated with worse 21 -day mortality (P-value 0.35 ). There are several plausible reasons explaining why NEWS is superior in predicting mortality. The greater number of variables monitored facilitates the detection of a physiological abnormality [9]. The SIRS sepsis criterion does not include blood pressure and lactate, both of which are valuable risk-stratification tools [22]. Furthermore the type of scoring system may have an influence on its sensitivity. NEWS scoring produces a continuum from $0-20$, whereas SIRS scoring is discrete, for which patients score 1 or 0 for each parameter based on whether the cut-off value is met. NEWS thus allows inferences to be made from the cumulative effect of deranged parameters even if they are below the specific cut-off value specified in the SIRS criteria. On the other hand, concerns have been expressed about NEWS because of its high sensitivity and exclusion of some parameters such as urine output [9].

Given the complexity and heterogeneity of sepsis, there may be no perfect scoring system for all situations, and experienced clinical judgement, although also not perfect [23], should be considered. Nevertheless there is a clear need to optimize our current definitions, to improve early recognition and appropriate management.

This study has a number of limitations. This was a preliminary, although painstakingly detailed analysis of consecutive cases, and the sample size was small. This compromised our ability to perform multivariate analysis. Nevertheless it was sufficient to generate some statistically significant results. Secondly this was a single centre study, potentially limiting its generalizability. Thirdly, we have not included qSOFA in our comparisons. This is partly because qSOFA was not developed on real-world data, and subsequent studies have suggested that it offers little for front-door assessment.

\section{Conclusion}

Septic shock is associated with a high 21-day mortality. The DiPS definition of Septic Shock was shown to capture more patients and to be a better predictor of mortality compared to the traditional SIRS definition. Larger prospective studies are needed for validation of the DiPS Septic Shock definition.

\section{Acknowledgements}

The authors would like to acknowledge the efforts of all the ED staff at the University Hospital Wales for their commitment to the project.

\section{Conflicts of Interest}

The authors declare that they have no conflicts of interest.

\section{Funding statement}

This research did not receive any specific grant from funding agencies in the public, commercial or not-for-profit sectors.

\section{References}

1. Mayr FB, Yende S, Angus DC (2014) Epidemiology of severe sepsis. Virulence 5: 4-11. [Crossref]
2. Szakmany T, Lundin R, Sharif B, Ellis G, Morgan P, et al. (2017) Sepsis prevalence and outcome on the general wards and emergency departments in wales: results of a multi-centre, observational, point prevalence study. PLOSone 11: e0167230. [Crossref]

3. The College of Emergency Medicine (2012) CEM clinical audits 2011-2012: severe sepsis and septic shock. College of Emergency Medicine, London.

4. Clinical Excellence Commission (2011) Quality Systems Assessment self-assessment Supplementary report-sepsis. Sydney: CEC, 2012.

5. Daniels R, Nutbeam T, McNamara G, Galvin C (2011) The sepsis six and the severe sepsis resuscitation bundle: a prospective observational cohort study. Emerg Med J 28: 507-512. [Crossref]

6. Burrell A, McLaws ML, Fullick M, Sullivan RB, Sindhusake D (2016) Sepsis kills: early intervention saves lives. Med J Aust 204: 1-7.

7. Singer M, Deutschman C, Seymour C, Shankar-Hari M, Annane D, et al. (2016) The third international consensus definitions for sepsis and septic shock. JAMA 315: 801810. [Crossref]

8. Shankar-Hari M1, Phillips GS2, Levy ML3, Seymour CW4, Liu VX5, et al. (2016) Developing a New Definition and Assessing New Clinical Criteria for Septic Shock: For the Third International Consensus Definitions for Sepsis and Septic Shock (Sepsis-3). JAMA 315: 775-787. [Crossref]

9. Morgan RJ, Williams F, Wright MM (1997) An early warning scoring system for detecting developing critical illness. Clin Intensive Care 8: 100.

10. McLymont N and Glover Guy W (2016) Scoring systems for the characterisation of sepsis and associated outcomes. Ann Transl Med 4: 527. [Crossref]

11. Kmietowicz Z (2015) Identify sepsis in patients by using early warning scores, doctors are urged. BMJ 351: h6237. [Crossref]

12. Serafim R, Gomes JA, Salluh J, Póvoa P (2018) A Comparison of the Quick-SOFA and Systemic Inflammatory Response Syndrome Criteria for the Diagnosis of Sepsis and Prediction of Mortality: A Systematic Review and Meta-Analysis. Chest 153: 646-655. [Crossref]

13. Rainer R, Li Y, Chan CP, Agarwal N, Sin K, et al. (2014) Validating a pragmatic definition of shock in adult patients presenting to the emergency department Resuscitation 85: S113.

14. Centers for Disease Control and Prevention (2017) Evaluation for Severe Sepsis Screening Tool.

15. http://apps.dataunitwales.gov.uk/wimd

16. Reinhart K, Daniels R, Kissoon N, Machado FR, Schachter RD, et al. (2017) Recognizing Sepsis as a Global Health Priority - A WHO Resolution. $N$ Engl J Med 377: 414-417. [Crossref]

17. Kang C, Song J, Chung D, Peck K, Ko K, et al. (2011) Risk factors and pathogenic significance of severe sepsis and septic shock in 2286 patients with gram-negative bacteremia. J Infect 62: 26-33

18. Esper AM, Moss M, Lewis CA, Nisbet R, Mannino DM, et al. (2006) The role of infection and comorbidity: Factors that influence disparities in sepsis. Crit Care Med 34: 2576-2582. [Crossref]

19. Houwink A, Rijkenberg S, Bosman R, van der Voort P (2016) The associations between lactate, mean arterial pressure, central venous oxygen saturation and peripheral temperature and mortality in severe sepsis: a retrospective cohort analysis. Critical Care 20: 1.

20. Goodhart I, Faulds M, Lobaz S, Glossop A (2015) Initial pH and mortality in patients with exacerbations of COPD and pneumonia treated with NIV in a teaching hospital critical care unit. Critical Care 19: 1.

21. Bilben B, Grandal L and Sovik S (2016) National early warning score (NEWS) as an emergency department predictor of disease severity and 90-day survival or disease severity and 90-day survival in the acutely dyspneic patient-a prospect observational study. Scand J Trauma Resusc Emerg Med 24: 80. [Crossref]

22. Shapiro NI, Howell MD, Talmor D, Nathanson LA, Lisbon A, et al. (2005) Serum lactate as a predictor of mortality in emergency department patients with infection. Ann Emerg Med 45: 524-528. [Crossref]

23. Li YL, Mo JR, Cheng NM, Chan SSW, Lin PY, et al. (2017) Rainer TH. Gestalt for shock and mortality in the emergency department: A prospective study. Am J Emerg Med 36: 988-992. [Crossref]

24. National Health Service [Internet] (2017). NHS Digital UK. A and E initial assessment triage category.

Copyright: (C2018 Walford RA. This is an open-access article distributed under the terms of the Creative Commons Attribution License, which permits unrestricted use, distribution, and reproduction in any medium, provided the original author and source are credited. 